\title{
Efficient Routing of Single Photons by One Atom and a Microtoroidal Cavity
}

\author{
Takao Aoki, ${ }^{1, *}$ A. S. Parkins, ${ }^{1, \dagger}$ D. J. Alton, ${ }^{1}$ C. A. Regal, ${ }^{1}$ Barak Dayan, ${ }^{1,}$ E. Ostby, ${ }^{2}$ K. J. Vahala, ${ }^{2}$ and H. J. Kimble ${ }^{1}$ \\ ${ }^{1}$ Norman Bridge Laboratory of Physics 12-33, California Institute of Technology, Pasadena, California 91125, USA \\ ${ }^{2}$ T. J. Watson Laboratory of Applied Physics, California Institute of Technology, Pasadena, California 91125, USA
}

(Received 8 October 2008; published 27 February 2009)

Single photons from a coherent input are efficiently redirected to a separate output by way of a fibercoupled microtoroidal cavity interacting with individual cesium atoms. By operating in an overcoupled regime for the input-output to a tapered fiber, our system functions as a quantum router with high efficiency for photon sorting. Single photons are reflected and excess photons transmitted, as confirmed by observations of photon antibunching (bunching) for the reflected (transmitted) light. Our photon router is robust against large variations of atomic position and input power, with the observed photon antibunching persisting for intracavity photon number $0.03 \leqq \bar{n} \lesssim 0.7$.

Cavity quantum electrodynamics (cQED) offers systems in which the coherent interaction between matter and light can dominate irreversible channels of dissipation [1-3]. Diverse systems based upon radiative interactions in cQED are thereby promising candidates for the physical implementation of quantum networks, where, for example, atoms in optical cavities (quantum nodes) are linked by photons in optical fiber (quantum channels) [3]. Although many important capabilities for quantum nodes have been demonstrated within the setting of CQED with single atoms in Fabry-Perot cavities [4-9], an outstanding challenge is high efficiency transport of quantum fields into and out of optical cavities [3], as is required to link large numbers of quantum nodes.

In this regard, the coupling rate $\kappa$ of photons to and from the quantum channel should dominate the rates for any other input-output mechanisms. One way to achieve this is to operate the nodes in an overcoupled regime [10], where external coupling dominates internal system losses.

In this Letter, we realize a cQED system in the optical domain with efficient input-output coupling while still maintaining high internal efficiency for coupling to a single atom. We use a microtoroidal cavity interacting with single cesium atoms [11-13], with coupling to and from the cavity implemented with a tapered optical fiber in an overcoupled regime [10]. We demonstrate an efficient and robust photon router for which single photons are extracted from an incident coherent state and redirected to a separate output with efficiency $\xi \simeq 0.6$.

To model photon transport for the atom-cavity system, we consider the interaction of one atom with the evanescent fields of a microtoroidal cavity, as shown in Fig. 1(a), with $g_{\text {tw }}$ (traveling wave) the rate of coherent atom-cavity coupling [14]. Near the atomic resonance at frequency $\omega_{A}$, the cavity supports two counterpropagating modes $a, b$ of frequency $\omega_{C}$. These cavity modes have intrinsic loss rate $\kappa_{i}$ and are coupled to each other at rate $h$ (e.g., due to internal scattering) [10]. Input to and output from the internal modes of the cavity are provided by a tapered fiber with external coupling rate $\kappa_{\mathrm{ex}}$, where the fields of the tapered fiber are denoted by $\left\{a_{\text {in }}, a_{\text {out }}, b_{\text {in }}, b_{\text {out }}\right\}$ in Fig. 1(a). For single-sided excitation as in our current experiment, the input modes have coherent amplitudes

$$
\left\langle a_{\mathrm{in}}\right\rangle=-\frac{i \mathcal{E}_{p} e^{-i \omega_{p} t}}{\sqrt{2 \kappa_{\mathrm{ex}}}}, \quad\left\langle b_{\mathrm{in}}\right\rangle=0 .
$$

The Hamiltonian, master equation, and input-output relations for the system depicted in Fig. 1(a) can be found in Refs. $[12,13,15]$. When the total cavity decay rate, $\kappa=$ $\kappa_{i}+\kappa_{\mathrm{ex}}$, is much larger than any other rate in the system,
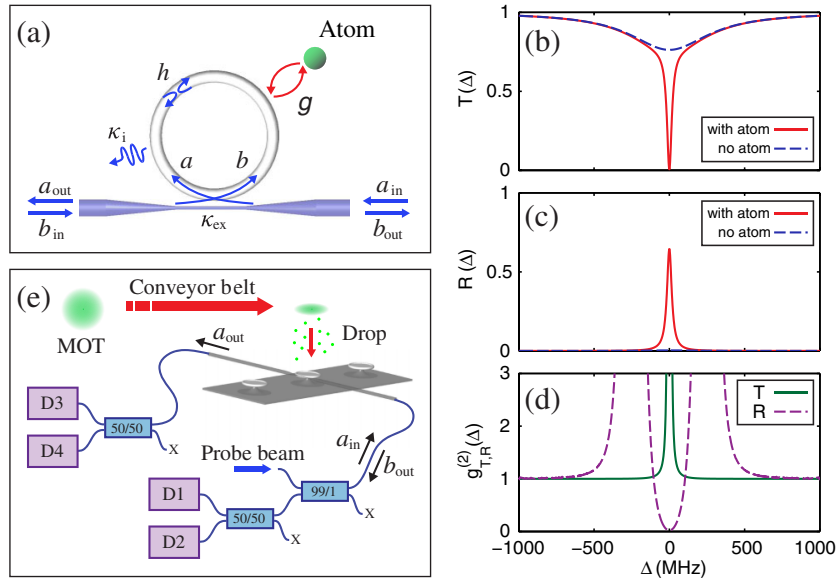

FIG. 1 (color online). (a) Simple depiction of one atom coupled to a toroidal cavity, together with fiber taper and relevant field modes, with rates $\left(g_{\mathrm{tw}}, \kappa_{\mathrm{ex}}, \kappa_{i}, h\right)$ as defined in the text. (b)-(d) Theoretical plots for the parameters of our experiment, $\left(g_{\text {tw }}, \kappa_{\text {ex }}, \kappa_{i}, h\right) / 2 \pi=(50,300,20,10) \mathrm{MHz}$, with $\omega_{A}=\omega_{C}$. (b), (c) Transmission and reflection spectra $T(\Delta), R(\Delta)$ for $a_{\text {out }}, b_{\text {out }}$ as functions of probe detuning $\Delta=\omega_{C}-\omega_{p}$ with and without the atom. (d) Theoretical intensity correlation functions versus $\Delta$ for the transmitted $\left[g_{T}^{(2)}(\tau=0)\right]$ and reflected $\left[g_{R}^{(2)}(\tau=0)\right]$ fields. (e) Schematic of our experiment. 
the cavity modes can be adiabatically eliminated from the system dynamics [16]. The system can then be described by effective optical Bloch equations for a two-level atom with the cavity-enhanced atomic decay rate $\Gamma=$ $\gamma(1+2 \mathcal{C})$, where $\mathcal{C}=\frac{2\left|g_{\mathrm{tw}}\right|^{2} \kappa}{\gamma\left(\kappa^{2}+h^{2}\right)}$ is the cooperativity parameter for a single atom and $\gamma$ is the rate of atomic decay to modes other than $a, b$.

In the overcoupled regime of toroid-taper coupling, $\kappa_{\text {ex }} \gg\left(\kappa_{i}, h\right)$, the analytic expressions for steady-state transmitted $I_{T}=\left\langle a_{\text {out }}^{\dagger} a_{\text {out }}\right\rangle_{\mathrm{ss}}$ and reflected $I_{R}=$ $\left\langle b_{\text {out }}^{\dagger} b_{\text {out }}\right\rangle_{\text {ss }}$ fluxes, and for the second-order correlation functions, $\quad g_{T}^{(2)}(\tau)=\left\langle a_{\text {out }}^{\dagger} a_{\text {out }}^{\dagger}(\tau) a_{\text {out }}(\tau) a_{\text {out }}\right\rangle_{\mathrm{ss}} / I_{T}^{2} \quad$ and $g_{R}^{(2)}(\tau)=\left\langle b_{\text {out }}^{\dagger} b_{\text {out }}^{\dagger}(\tau) b_{\text {out }}(\tau) b_{\text {out }}\right\rangle_{\text {ss }} / I_{R}^{2}$, can be greatly simplified. Specifically, at zero detuning (i.e., $\omega_{A}=\omega_{C}=$ $\left.\omega_{p}\right)$ and in the weak driving limit, the transmission and reflection coefficients are given by [15]

$$
\begin{gathered}
T(\Delta=0) \equiv I_{T}(0) / I_{T}(\Delta \gg \kappa) \equiv T_{0} \simeq \frac{1}{(2 \mathcal{C}+1)^{2}}, \\
R(\Delta=0) \equiv I_{R}(0) / I_{T}(\Delta \gg \kappa) \equiv R_{0} \simeq\left(\frac{2 \mathcal{C}}{2 \mathcal{C}+1}\right)^{2},
\end{gathered}
$$

while second-order intensity correlations at zero time delay are found to be

$$
\begin{gathered}
g_{T}^{(2)}(\tau=0) \simeq\left(4 \mathcal{C}^{2}-1\right)^{2}, \\
g_{R}^{(2)}(\tau=0) \simeq 0 .
\end{gathered}
$$

The physical interpretation of these results is as follows. In steady state and with $\mathcal{C} \gg 1$, the field radiated by the atomic polarization $\sigma_{-}^{\text {SS }}$ interferes destructively with the intracavity field from $a_{\text {in }}$, resulting in small transmission $T_{0} \ll 1$. But $\sigma_{-}^{\text {SS }}$ also coherently drives the intracavity field $b$, thereby generating a backward propagating field $b_{\text {out }}$, leading to reflection $R_{0} \rightarrow 1$ [17]. On the other hand, the overcoupled cavity without the atomic polarization (i.e., $\mathcal{C}=0$ ) has $T_{0} \simeq 1$ and $R_{0} \simeq 0$. Strong optical nonlinearity of the atom-toroid system gives rise to a dynamical switching between these two limits conditioned upon photon detection. (1) $T_{0} \simeq 0, R_{0} \simeq 1$ due to the field from $\sigma_{-}^{\mathrm{SS}}$, and (2) $T_{0} \simeq 1, R_{0} \simeq 0$ due to the conditional atomic polarization $\sigma_{-}^{C}$ following photon detection [18].

This description is substantiated by examination of $g_{T, R}^{(2)}$. Detection of a "first" photon for the reflected field $b_{\text {out }}$ projects the atom to the ground state, which precludes the detection of a "second" photon for $b_{\text {out }}[13,18]$. Hence, the reflected light exhibits sub-Poissonian photon statistics and photon antibunching. Similarly, photon detection for the transmitted light $a_{\text {out }}$ results in atomic projection that depends on $\mathcal{E}_{p}$ and $\mathcal{C}$ [18], with then $a_{\text {out }}$ displaying photon bunching and super-Poissonian statistics. The net effect is the routing of single photons into the backward direction $b_{\text {out }}$ and remaining excess photons into the forward field $a_{\text {out }}$.

Figures 1(b)-1(d) present theoretical results for transmission and reflection spectra $T(\Delta), R(\Delta)$ and intensity correlations $g_{T}^{(2)}(0), g_{R}^{(2)}(0)$ in the weak-driving limit for the parameters of our experiment: $\left(g_{\mathrm{tw}}, \kappa_{\mathrm{ex}}, \kappa_{i}, h\right) / 2 \pi=$ $(50,300,20,10) \mathrm{MHz}$. Although not deeply in the overcoupled regime, efficient transmission and reflection can be achieved, with $T_{0} \approx 0.005, R_{0} \approx 0.7$ with an intracavity atom, and $T_{0} \approx 0.8, R_{0} \approx 0.003$ without the atom.

A schematic of our experiment is shown in Fig. 1(e) and is similar to that described in Refs. [12,13]. However, our new apparatus has two major improvements that allow us to reproducibly tune $\kappa_{\text {ex }}$ to the desired overcoupled value and to maintain a small $\kappa_{i}$. First, the silicon chip supporting toroids and the tapered fiber are mounted on piezoelectric-driven stages that are placed inside the main chamber. The combination of compact, rigid design and good passive vibration-isolation enables us to tune the separation between the taper and toroid and to achieve stable coupling between the evanescent fields of the taper and toroid without having the taper contact the surface of the toroid. Second, in order to avoid degradation of quality factor for the toroids caused by cesium contamination, our new apparatus has a separate "MOT chamber" where cesium atoms are magnetooptically cooled and trapped, then loaded into an optical conveyor belt [19] and transferred into the "cQED chamber" through a differential pumping tube and positioned directly above a particular toroid on the silicon chip.

The resonance frequency $\omega_{C}$ of the toroid is tuned via the chip temperature near the frequency $\omega_{A}$ of the $6 S_{1 / 2}$, $F=4 \rightarrow 6 P_{3 / 2}, F^{\prime}=5$ transition of cesium, with $\mid \omega_{A}-$ $\omega_{C} \mathrm{l} / 2 \pi \lessgtr 30 \mathrm{MHz}$. The frequency $\omega_{p}$ of the probe beam $\mathcal{E}_{p}$ is locked to coincide with $\omega_{A}$, with $\left|\omega_{A}-\omega_{p}\right| / 2 \pi \lesssim$ $1 \mathrm{MHz}$. The probe is sent into the taper via the $1 \%$ port of 99/1 fiber beam splitter [Fig. 1(e)]. The 99\% port of this beam splitter is connected to a 50/50 beam splitter followed by two single-photon counting-modules (SPCMs), $D_{1}, D_{2}$, to detect the reflected field $b_{\text {out }}$. Similarly, the transmitted field $a_{\text {out }}$ in the taper is detected with another pair of SPCMs, $D_{3}, D_{4}$. Photoelectric events from the four SPCMs are time stamped and recorded for analysis.

The physical properties of the new toroids for our current experiment are similar to those in Ref. [13]. The major diameter $D \approx 25 \mu \mathrm{m}$ and minor diameter $d \approx 6 \mu \mathrm{m}$, leading to an effective atom-cavity coupling rate of $g_{\text {eff }} / 2 \pi \approx 70 \mathrm{MHz}\left(g_{\text {tw }} / 2 \pi \approx 50 \mathrm{MHz}\right)[12,14]$. Combined with the measured values for $\left(\kappa_{\mathrm{ex}}, \kappa_{i}, h\right)$ and with $\gamma / 2 \pi=5.2 \mathrm{MHz}$, we calculate the single-atom cooperativity parameter $\mathcal{C}=3.0$ and the cavity-enhanced atomic decay rate $\Gamma / 2 \pi=36.4 \mathrm{MHz}$. Note that for large toroidtaper separation with $\kappa_{\mathrm{ex}} \simeq \kappa_{i}$, our system is in the strong coupling regime of $\mathrm{cQED}$ with $g_{\text {eff }}>\kappa, \gamma$.

About $10^{7}$ atoms with temperature $T \approx 100 \mu \mathrm{K}$ are dropped from a height of $0.5 \mathrm{~mm}$ above the toroid by turning off the dipole trap beams. As shown in Fig. 1(c), the reflected intensity $I_{R}$ associated with $b_{\text {out }}$ should be near zero when there is no atom in the cavity. A falling atom that interacts with the evanescent fields of the cavity 
generates an increase in $I_{R}$ for the duration of the atomic transit and enables single transit events to be observed with high signal-to-noise ratio [20]. While transiting atoms are sufficient for these experiments due to our insensitivity to atomic position; in the future, we recognize the need to trap and localize single atoms near the toroid to allow continuous operation of the system [6-9].

We first fix the probe power $\left|\mathcal{E}_{p}\right|^{2}$ such that the intracavity photon number $\bar{n}=0.093$ for the empty cavity. Atom transit events are extracted from the records of photoelectric counts $C_{1,2}(t)$ from $b_{\text {out }}$ at detectors $D_{1}, D_{2}$ by the following procedure. For an atom transit event, we require the sum of the counts over a time $\Delta t_{\text {atom }}, S_{j} \equiv$ $\sum_{t_{i}=t_{j}}^{t_{j}+\Delta t_{\text {tom }}}\left[C_{1}\left(t_{i}\right)+C_{2}\left(t_{i}\right)\right]$, to be equal or greater than a threshold count $C_{\mathrm{th}}$. After applying this selection criterion to $C_{1,2}(t)$, we determine the time origin $t=0$ for each transit event by the temporal mean of the counts in $\Delta t_{\text {atom }}$ and extract counts in a time window of $\pm 6 \mu \mathrm{s}$ around $t=0$ for further analysis.

Figures 2(a) and 2(b) show the transmitted $\mathcal{T}_{0}(t)$ and reflected $\mathcal{R}_{0}(t)$ signals averaged over transit events with transit selection criteria of $C_{\text {th }}=4,5,6$ and $\Delta t_{\text {atom }}=$ $4 \mu \mathrm{s}$, where $\mathcal{T}_{0}(t)$ and $\mathcal{R}_{0}(t)$ are normalized to the resonant transmission of the empty cavity, $T_{0} \simeq 0.83$. Atomcavity coupling results in reduced transmission and increased reflection [21]. The second-order correlation functions $g_{T R R}^{(2)}(\tau)$ for the transmitted and reflected fields $a_{\text {out }}$ are calculated in the same manner as in Refs. [13,15] and plotted in Figs. 2(c) and 2(d). Photon bunching and antibunching are clearly observed for $a_{\text {out }}, b_{\text {out }}$ in the forward and backward directions, respectively. The recovery time $\tau_{R}$ to steady state is set by the cavity-enhanced atomic decay rate $\Gamma=\gamma(1+2 \mathcal{C})$, with $\tau_{R} \sim 1 / \Gamma[13,15]$.
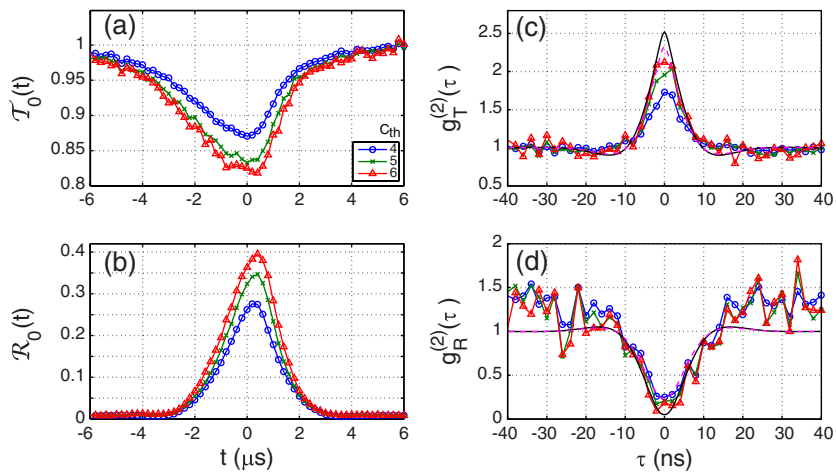

FIG. 2 (color online). (a), (b) Average atom transit signals for (a) transmission $\mathcal{T}_{0}(t)$ and (b) reflection $\mathcal{R}_{0}(t)$ of the probe field. As shown in the inset in (a), the transit selection criteria are set to be $C_{\mathrm{th}}=4,5,6$, where in all cases, $\Delta t_{\text {atom }}=4 \mu \mathrm{s}$. (c), (d) The intensity correlation functions $g_{T, R}^{(2)}(\tau)$ for the transmitted field $a_{\text {out }}$ and the reflected field $b_{\text {out }}$. For (a)-(d), $\bar{n}=0.093$ photons. Solid lines are a theoretical calculation using the parameters $\left(g_{\text {tw }}, \kappa_{\text {ex }}, \kappa_{i}, h\right) / 2 \pi=(50,300,20,10) \mathrm{MHz}$. Dashed lines are the same calculation with $4 \%$ background counts.
These observations are consistent with the theoretical predictions in Figs. 1(b) -1 (d) for $\Delta=0$, thereby demonstrating a functional photon router. Note that the traces with larger threshold $C_{\text {th }}$ show more significant effects of atom-cavity coupling. This is because we have fewer "falsely detected transits" (i.e., time windows that have no atom but nevertheless satisfy the selection criterion $S_{j} \geq C_{\text {th }}$ because of background light) and reduced contributions from transits with smaller $g$.

To explore this point further, in Fig. 3(a), we plot the ratio $F$ of false to total atomic transit events, $F \equiv \mathcal{R}_{0}^{\text {no }}(t=$ $0) / \mathcal{R}_{0}(t=0)$, where $\mathcal{R}_{0}^{\text {no }}$ is the measured transit signal for the reflected field as in Fig. 2(b), but now with no atoms present. Clearly, $F$ decreases as $C_{\text {th }}$ increases. Correspondingly, in 3(b)-3(d), $\mathcal{T}_{0}, g_{T}^{(2)}$, and $g_{R}^{(2)}$ show more significant effects of atom-cavity coupling with increasing $C_{\mathrm{th}}$, albeit with larger error bars due to the diminishing number of transit events. The value $C_{\mathrm{th}}=5(F \simeq 0.025)$ provides a reasonable compromise between decreasing signal-tonoise ratio and reduced contamination from false transit events.

Finally, in order to investigate the saturation behavior of our photon router, we vary the intracavity photon number by way of the probe intensity $\left|\mathcal{E}_{p}\right|^{2}$. Figure 4 displays the measured values of $\mathcal{T}_{0}(t=0)$ and $g_{R}^{(2)}(\tau=0)$ as functions of $\bar{n}$. For each value of $\bar{n}$, we set the selection threshold $C_{\text {th }}$ so that the false detection ratio $F<0.05$. The point for the lowest value of $\bar{n}$ for $g_{R}^{(2)}(\tau=0)$ is omitted from (b) due to its poor signal-to-noise ratio. In (a), the transmission $\mathcal{T}_{0}(t=0)$ shows clear saturation of the atom-cavity coupling, from $\mathcal{T}_{0} \approx 0.2$ at $\bar{n} \approx 0.01$ to $\mathcal{T}_{0} \approx 0.8$ at $\bar{n} \approx 0.7$. Importantly, in (b) $g_{R}^{(2)}(\tau=0)<1$ over a wide range of intracavity photon number, $0.01 \lesssim \bar{n} \lesssim 0.7$.

From the measured $\mathcal{T}_{0}$ and known parameters $\left(g_{\mathrm{tw}}, \kappa_{\mathrm{ex}}\right.$, $\left.\kappa_{i}, h\right)$, we estimate the efficiency $\xi$ for single-photon throughput and obtain $\xi \approx 0.6$ for the lowest $\bar{n} \approx 0.012$,
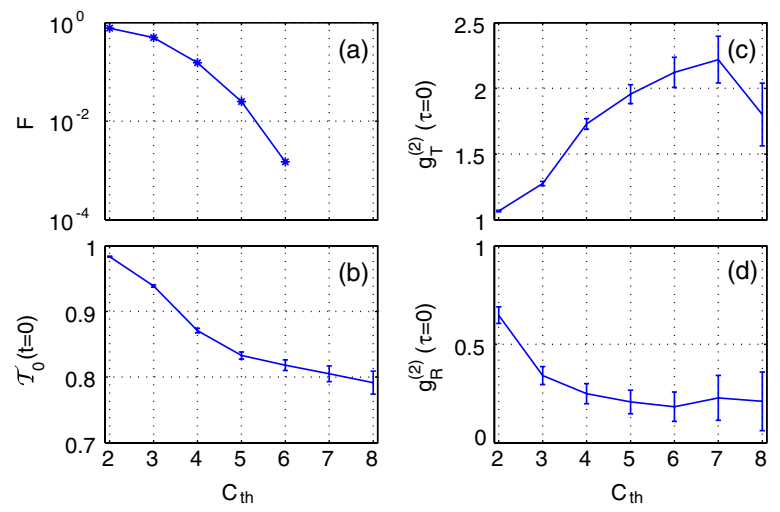

FIG. 3 (color online). (a) False detection ratio $F$, (b) transmitted signal $\mathcal{T}_{0}(t=0)$ at the center of an atomic transit, and (c), (d) intensity correlation functions $g_{T, R}^{(2)}(\tau=0)$ at zero time delay for the transmitted $T$ and reflected $R$ light as functions of the threshold $C_{\text {th }}$ for the selection of atom transits. In all cases, $\Delta t_{\text {atom }}=4 \mu \mathrm{s}$ and $\bar{n}=0.093$. 

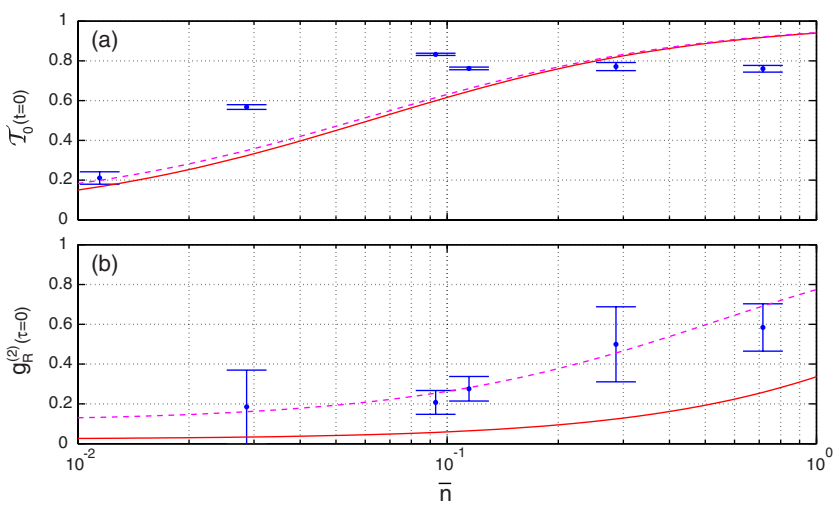

FIG. 4 (color online). (a) Transmitted signal $\mathcal{T}_{0}(t=0)$ at the center of an atomic transit and (b) intensity correlation function $g_{R}^{(2)}(\tau=0)$ at zero time delay for the reflected light for various values of intracavity photon number $\bar{n}$. Points are experimental data averaged over individual transit events. Solid lines are from a theoretical calculation with the parameters $\left(g_{\mathrm{tw}}^{\min }, g_{\mathrm{tw}}^{\max }, \kappa_{\mathrm{ex}}, \kappa_{i}, h\right) / 2 \pi=(35,65,300,20,10) \mathrm{MHz} \quad$ where instead of a single value of $g_{\mathrm{tw}}$, we use an average over $g_{\mathrm{tw}}^{\min }$ to $g_{\mathrm{tw}}^{\max }$. Dashed lines are the same calculation, but with the assumption of background counts of $4 \%$ of the signal.

with $\xi \rightarrow 0.7$ for $\bar{n} \rightarrow 0$. This is consistent with the value $\xi \approx 0.5 \pm 0.2$ directly inferred from the distribution of $\mathcal{R}_{0}$ for the individual transits. As evidenced in Figs. 2(a) and 2(c), multiple photons are efficiently transmitted into the output channel $a_{\text {out }}$.

The solid lines in Fig. 4 are from a simplified theoretical model [15]. The curves are obtained from an average over the atomic azimuthal position $k x$ around the major circumference of the toroid. Curves for particular values of $k x$ would be indistinguishable on the scale of the plot. This insensitivity to $k x$ is due to the small coupling between the counterpropagating modes $a$ and $b$, characterized by the ratio $h / \kappa \simeq 0.03 \ll 1$, that limits the formation of a standing wave [15].

To account for background light, the dashed lines in Fig. 4 include background counts at a level of $4 \%$ of the signal. This leads to good agreement for the backgroundsensitive parameter $g_{R}^{(2)}(\tau=0)$ in Fig. 4(b). There still remains a discrepancy for $\mathcal{T}_{0}$ in Fig. 4 (a) given the statistical error bars, even when we account for variation in $g_{\text {tw }}$ by averaging over a range of values around $g_{\mathrm{tw}}=50 \mathrm{MHz}$ $\left(g_{\mathrm{tw}}=35 \mathrm{MHz}\right.$ to $\left.g_{\mathrm{tw}}=65 \mathrm{MHz}\right)$. Thus, we must attribute the discrepancy in (a) to a systematic uncertainty in the experiment.

In summary, we have realized a photon router using single cesium atoms coupled to a microtoroidal cavity in the overcoupled regime. In comparison to our previous photon turnstile [13], this photon router is robust against azimuthal position of the atom $(k x)$ and taper-toroid distance $\left(\kappa_{\mathrm{ex}}\right)$, in addition to the atom-cavity coupling strength $(g(\vec{r}))$. The current system already has throughput efficiency for single photons of $\xi \approx 0.6$, and it is projected to reach $\xi>0.999$ through use of smaller toroid diameter [22] and improvement of the intrinsic quality factor of the cavity to $Q \approx 10^{10}$ [23]. The realization of strong interactions of single photons and atoms together with efficient input-output provides an enabling capability for the realization of quantum networks and investigations of quantum many-body systems built component by component.

We gratefully acknowledge the contributions of $\mathrm{S}$. Kelber and E. Wilcut-Connolly. This work is supported by the NSF PHY-0652914, by IARPA via ARO, and by NGST. A.S.P. thanks the Marsden Fund of the Royal Society of New Zealand for support.

*Permanent addresses: Department of Physics, Kyoto University, Kyoto, Japan, and PRESTO, Japan Science and Technology Agency, Saitama, Japan.

†epartment of Physics, University of Auckland, Auckland, New Zealand.

*Department of Chemical Physics, Weizmann Institute of Science, Rehovot, Israel.

[1] K. J. Vahala, Nature (London) 424, 839 (2003).

[2] G. Khitrova et al., Nature Phys. 2, 81 (2006).

[3] H. J. Kimble, Nature (London) 453, 1023 (2008).

[4] Q. A. Turchette et al., Phys. Rev. Lett. 75, 4710 (1995).

[5] T. Wilk et al., Science 317, 488 (2007).

[6] J. McKeever et al., Science 303, 1992 (2004).

[7] M. Hijelkema et al., Nature Phys. 3, 253 (2007).

[8] M. Keller et al., Nature (London) 431, 1075 (2004).

[9] A. D. Boozer et al., Phys. Rev. Lett. 98, 193601 (2007).

[10] S. M. Spillane et al., Phys. Rev. Lett. 91, 043902 (2003).

[11] D. K. Armani et al., Nature (London) 421, 925 (2003).

[12] T. Aoki et al., Nature (London) 443, 671 (2006).

[13] B. Dayan et al., Science 319, 1062 (2008).

[14] The rate of coherent interaction between the atom and the modes $a, b$ is given by $g_{\mathrm{tw}}=g_{0}^{\mathrm{tw}} f(\rho, z) e^{ \pm i k x}$, where $\rho$ is the atom-toroid radial distance, $x$ is the atom's position around the toroid circumference, and $z$ is the vertical coordinate.

[15] Supporting Online Material accompanying Ref. [13].

[16] C. W. Gardiner and P. Zoller, Quantum Noise (SpringerVerlag, Berlin, 1999), 2nd ed.

[17] J. T. Shen and S. Fan, Opt. Lett. 30, 2001 (2005).

[18] H. J. Carmichael, Phys. Rev. Lett. 70, 2273 (1993).

[19] S. Kuhr et al., Science 293, 278 (2001).

[20] For our previous work [12,13], the taper and toroid were critically coupled with the taper in contact mode. Atom transits were detected as increases in the transmitted intensity $I_{T}$ for the field $a_{\text {out }}$.

[21] The asymmetry evident in Fig. 2(a) and 2(b) is a robust feature of our observations. We have no definitive explanation, but are investigating various possible causes, including surface forces.

[22] S. M. Spillane et al., Phys. Rev. A 71, 013817 (2005).

[23] D. W. Vernooy et al., Opt. Lett. 23, 247 (1998). 